\title{
Le sacré affleure-t-il aussi au musée ?
}

Does the sacred blossom in the museum?

\section{Fanny Fouché}

\section{(2) OpenEdition}

\section{Journals}

Édition électronique

URL : http://journals.openedition.org/iss/1570

ISSN : 2306-4161

\section{Éditeur}

ICOM - International Council of Museums

Édition imprimée

Date de publication : 12 octobre 2019

Pagination : 105-121

ISBN : 978-92-9012-467-2

ISSN : 2309-1290

\section{Référence électronique}

Fanny Fouché, «Le sacré affleure-t-il aussi au musée ? », ICOFOM Study Series [En ligne], 47(1-2) |

2019, mis en ligne le 12 octobre 2019, consulté le 24 octobre 2019. URL : http://

journals.openedition.org/iss/1570 


\title{
Le sacré affleure-t-il aussi au musée ?1
}

\author{
Fanny Fouché \\ Ecole du Louvre - Paris, France \\ Université de Neuchâtel - Neuchâtel, Suisse
}

La Nature est un temple où de vivants piliers

Laissent parfois sortir de confuses paroles L'homme y passe à travers des forêts de symboles

Qui l'observent avec des regards familiers

Comme de longs échos qui de loin se confondent

Dans une ténébreuse et profonde unité

Vaste comme la nuit et comme la clarté

Les parfums, les couleurs et les sons se répondent

Charles Baudelaire, Correspondances, Les Fleurs du Mal

ABStRACT

\section{Does the sacred blossom in the museum?}

Western Museums have composed themselves from fragments born in different cultures, integrated into the matrix of their own exhibition logic. This appropriation thus constitutes a confrontation between the competing values that objects have inherited in systems from which they came, and the values upon which the museum institution itself

I. Ce titre fait écho au titre d'une conférence de Brigitte d'Hainaut-Zvény intitulée « Des retables et des hommes ou comment faire affleurer une présence du sacré dans les sanctuaires (XIII ${ }^{e}-X V I I I^{e}$ ) » (Académie Royale des Sciences, des Lettres et des Beaux-Arts de Belgique, avril 20I5). 
was built. As an entity on this border between immediate presence and connective transcendence, the altarpiece is proposed here as a locus to observe the dynamic process of sacralization. Finally, the journey of the altarpiece from the ecclesiastical place interrogates, on a theoretical level, the transfer of sacredness at work in its displacement.

Keywords: altarpieces, sacralization, hierophanie, sacred/profane space, museum, transfer

\section{RÉSUMÉ}

Le musée occidental procède par incorporation de fragments issus de cultures multiples qu'il intègre dans la matrice de sa propre logique d'exposition. Cette appropriation constitue une confrontation entre les valeurs concurrentes dont les objets étaient porteurs dans les systèmes dont ils étaient issus et celles structurant l'institution muséale elle-même. Cet article approche le retable comme un objet dont la situation initiale, à la frontière avec la transcendance, permet de questionner les processus au cœur de la sacralisation. Les pérégrinations du retable depuis l'espace ecclésial invitent à envisager le transfert de sacralité à l'œuvre dans son déplacement.

Mots clé : retables, sacralisation, hiérophanie, espace sacré/profane, musée, transfert

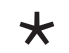

\section{Introduction}

L'enveloppement du sanctuaire et les dédales du musée n'évoquent-ils pas ces temples aux vivants piliers entre lesquels des sons indistincts s'élèvent comme des murmures? L'homme ne s'y avance-t-il pas entre des forêts de symboles qui, tandis qu'il les contemple, l'observent avec des regards familiers? Les temples de la nature, de la foi et de la connaissance auraient-ils en commun de lui offrir d'entrevoir son lien avec ceux qui l'ont précédé comme avec les choses qui l'entourent? L'intuition d'une proximité entre le musée et le temple a-t-elle quelque fondement? Les fragments de culture que le musée rassemble et protège font-ils encore entendre en son sein les «correspondances » et les échos dont ils furent jadis porteurs? Ayant permis des rapprochements féconds entre la muséologie et le sacré, le symposium de Téhéran invite à pousser plus loin le questionnement. Entre l'espace sacré et l'espace muséal, entre le pèlerinage religieux et le tourisme culturel, entre la gestuelle liturgique et la gestion patrimoniale, ou encore entre la vénération religieuse et l'admiration artistique (Mairesse, 2014), y a-t-il apparence de ressemblance ou communauté 
de nature ? Le sacré est-il une dimension intrinsèque à l'essence du musée ? Y entre-t-il par contact avec les objets sacrés au cour de ses collections? Est-ce au contraire une qualité de ces artefacts qui s'évaporerait au franchissement du seuil muséal ? S'agirait-il enfin d'une dimension immatérielle que le musée ingérerait en même temps que les objets qui en étaient porteurs ${ }^{1}$ ?

Le voyage qui conduit le retable² de l'espace ecclésial aux cimaises d'un musée - entendu ici dans son acception façonnée par les Lumières de temple de l'histoire de l'art et conservatoire des civilisations - offre un observatoire idéal pour aborder l'hypothèse d'un transfert de sacralité d'un univers à l'autre. Dans cette perspective, le texte "The nature of numinosity and its museological reconstruction" ouvre magistralement la voie. Michael Ames (1994/1995) y montre le caractère construit sociohistoriquement de la sacralité et combien celle-ci relève d'une élaboration complexe à la fois dans le milieu dont proviennent les objets et dans le musée. Etablissant que « la re-représentation d'un objet dans l'enceinte muséale n'est pas plus artificielle que sa représentation première », Ames (1994/1995, p. 6I) donne la marche à suivre. Il invite à se pencher sur la façon dont le christianisme, ici au cours du Moyen Age, fait du retable le siège d'une rencontre possible entre immanence et transcendance. Sa démarche implique de clarifier l'existence de processus dynamiques contribuant à façonner la dimension sacrée jadis attachée à ce type d'objet. L'espace de la collection Renaissance et celui du musée actuel deviennent alors des laboratoires au sein desquels examiner la réapparition de procédés jouant des ressorts de la sacralisation.

\section{Le sacré : une expérience plus qu'un concept}

Questionner «la sacralité » au musée, qu'elle s'attache aux objets, à leur interprétation ou à la logique muséale elle-même, implique de s'accorder sur ce qu'on entend par ce terme.

\section{Caractériser étymologiquement le sacré 3}

La notion de «sacré » a trait à l'idée d'un jaillissement, d'une manifestation à laquelle se confronte l'homme, quelle que soit la culture dans laquelle il vit cette expérience. Le grec hagnos montre que s'y attachent les sentiments contraires de crainte, d'effroi sacré et de vénération face à la majesté divine. Frisson et fascination situent dans le vécu humain le plus intime les ébranlements ressentis (Otto, 1968). C'est qu'il s'agit au départ de l'irruption de ce que Mircea Eliade a décrit comme " "ganz andere", radicalement et totalement différent : ne ressemblant à rien d'humain ou de cosmique » (Eliade, 1965,

\footnotetext{
I. Sur ce thème, voir (Gonseth, Hainard \& Kaehr, 2002).

2. Elément fixe ou mobile le plus souvent sculpté, orfévré et/ou peint constituant la partie postérieure de l'autel chrétien ou posé sur la table de celui-ci.

3. On suit ici le fil du moine bénédictin de l'abbaye de Münsterschwarzach, Anselm Grün (2004) dans ses méditations philosophiques sur le sacré.
} 
p. I6). Ce peut être la force mystérieuse désignée par les Mélanésiens comme mana. L'irruption de cette puissance hors de la sphère de la raison l'entoure d'exceptionnel, mais aussi de danger. On approche ici l'intouchable que désigne le tabou des Polynésiens affectant les points directs de contact avec le sacré. L'étymologie du terme en hébreux, qadosh, souligne l'idée de séparation dont l'archétype correspond, dans le récit biblique, à la confrontation de Moïse avec la terre sainte et emplie de Dieu où lui apparait le Buisson ardent. La distinction entre cet espace chargé de forces et sa périphérie, sans structure ni consistance, est cristallisée dans l'interdiction pour Moïse de souiller cette terre sainte du contact de sa sandale [Ex 3, 5]. L'importance de ces lieux et du respect de leur accès à travers des rituels d'approche précis se retrouve dans l'étymologie latine sacer. Les Romains désignaient en effet comme sanctus ces épicentres définis par les gestes consistant à les circonscrire. L'érection d'une frontière avec le profane - situé devant (pro-) le lieu sacré (-fanum) - définit ainsi l'espace sacré par sa position à l'écart. L'inaccessibilité de lieux érigés en « centres du monde » (Eliade, 1965), gardés de toute violation et ne pouvant être atteints qu'à travers des conditions et des rituels très précis, constitue donc une des dimensions intrinsèques à la caractérisation sacrale.

\section{De la manifestation du sacré}

La capacité de l'objet sacré à devenir le lieu d'un jaillissement est également primordiale. Sans doute est-ce l'étude de Mircea Eliade des spécificités de l'expérience religieuse qui éclaire le mieux cette dimension. Pour désigner l'irruption de la transcendance par une brèche dans le continuum du quotidien, ce dernier fonde la notion de hiérophanie. Il la désigne comme :

La manifestation de quelque chose de «tout autre », d'une réalité qui n'appartient pas à notre monde, dans des objets qui font partie intégrante de notre monde "naturel ", "profane " (Eliade, 1965, p. 17).

On perçoit clairement ici, et ce point a toute son importance dans la perspective qui nous intéresse, comment le sacré apparaît toujours à travers quelque chose d'audible, de visible, de palpable transfiguré par la réalité invisible qui soudain le traverse. Que ce soit dans une pierre, un arbre, une terre, un homme, "le sacré se manifeste au moyen de quelque chose d'autre que lui-même : êtres, objets, mythes ou symboles » (Ries, I986, p. 333). Le paradoxe originel de la hiérophanie se joue dans cette possibilité pour l'homme de se lier à des réalités terrestres, objets, lieux ou êtres médiateurs momentanément revêtus de cette dimension de sacralité qui les transforme. Il en résulte la possibilité d'une discontinuité fondamentale non seulement dans l'espace, mais également dans le cours du temps autour de l'expérience du sacré. A la conscience de l'existence d'espaces qualitativement différents d'autres répond la distinction 
entre le temps profane du quotidien et le temps sacré lié à la célébration des mythes originels. Si la sacralité s'élabore constitutivement par la qualification spatiale et temporelle (Dupront, 1987), elle s'appuie également sur les rites qui constituent son troisième instrument de prédilection pour ouvrir un passage du monde terrestre vers le monde céleste. Brosser à grands traits ces caractéristiques s'attachant de façon générique aux processus de sacralisation aidera-t-il à en reconnaître certains dans le façonnement de la numinosité des objets sacrés dans leur contexte d'apparition? S'avèrera-t-elle susceptible d'éclairer également des processus à l'œuvre au-delà de ces énonciations premières, dans des réinterprétations ultérieures de ces artefacts?

\section{Du retable sur l'autel au chef-d'œuvre sur la cimaise}

\section{Incipit : un objet d'art en suspension}

L'auréole de gloire entourant La Vierge aux rochers de Vinci offre un incipit à la réflexion. Ce joyau est si étroitement associé au prestige de la Grande Galerie du Louvre où il trône qu'il en devient difficile à transposer mentalement dans la chapelle de l'église San Francesco Grande à Milan à laquelle il fut d'abord destinét. C'est qu'avant de devenir le tableau dont l'histoire du Louvre signale la présence dans les collections royales françaises au moins depuis le milieu du XVII ${ }^{e}$ siècle, il fut le fragment central d'un polyptyque que la Confraternité de l'Immaculée Conception avait commandé à Léonard. Si de «longs échos qui parfois se confondent » peuvent par instant sourdre dans l'espace muséal, c'est bien qu'avant de devenir musealia, d'autres réalités, d'autres pratiques et faisceaux de représentations en ont constitué la raison et déterminé les choix esthétiques. Des espérances, des gestuelles d'oraison, des pratiques rituelles et des performances liturgiques ont façonné à la fois la forme matérielle et l'efficacité de bien des objets reconnus comme sacrés par d'autres sociétés ou d'autres temporalités. Peut-on encore entrevoir ces gestes et entendre ces voix dans l'espace muséal ? Aussi sûrement qu'« un crucifix roman n'était pas d'abord une sculpture » (Malraux, I949, p. I4) un retable n'était pas d'abord un tableau. Qu'était-il alors?

L'immense variété recouverte par le terme de retable au fil du temps et des orientations géographiques rend caduque l'idée d'en faire le tour en quelques pages (Van Os, I988/1990 ; Bousquet, 1982 ; Laclotte \& Thiébaud, 1990 ; Boskovits, 1992 ; Chastel, 1993 ; Gardner, 1994 ; Fuchss, 1999). Du devant d'autel des premiers siècles du christianisme, à la monumentalité des retables baroques, en passant par les retables sculptés de l'âge roman, ceux peints par exemple au XIII siècle ou encore les polyptyques à volets mêlant, au-delà du XV siècle, sculptures et représentations peintes, l'éventail des formes prises par le retable est considérable. Est-il au moins possible de jeter un peu de lumière sur la

\footnotetext{
I. On sait néanmoins qu'il n'y prit jamais place, une seconde version ayant emporté l'adhésion des commanditaires (celle conservée à la National Gallery de Londres).
} 
destinée muséale d'un tel objet ? On propose de s'y essayer en interrogeant d'abord la façon dont il fut investi dans le système religieux chrétien. Cet investissement a largement à voir avec le statut des images et des reliques.

Comme l'indique l'étymologie (retro-tabulae), le retable a partie liée avec l'autel chrétien et avec les reliques qu'il enchâsse. Il importe dès lors de l'appréhender en ayant à l'esprit la logique rituelle évoquée plus haut à travers laquelle certaines réalités terrestres, itinéraires, gestes ou objets se muent en puissances d'ouverture vers le ciel. En effet, les voies d'efficacité des reliques rappellent la façon dont la pierre, le cierge, l'huile, le signe de croix ou la génuflexion deviennent preuves en même temps que mémoriaux de la présence divine (Grün, 2004). Il faut dès lors se souvenir combien les reliquaires accentuent encore le caractère indiciel de ces réalités transitives puisque tout en lui tend « moins à montrer qu'à dévoiler, c'est-à-dire à cacher pour suggérer seulement et à ne révéler que de manière exceptionnelle » (Schmitt, I997, p. I59). Or, bien avant que s'y dressent les moindres prémices de retables, la sacralité de la table de la dernière Cène venait précisément de la présence des fragments de corps vénérés en son sein dictant l'interdiction, pendant les huit premiers siècles du christianisme, d'y déposer le moindre objet, en dehors du livre des Evangiles et de la custode eucharistique portant les saintes espèces. Envisager une histoire des retables remontant au décret pontifical accordant, autour du IX ${ }^{\mathrm{e}}$ siècle, aux reliquaires le privilège de trôner sur les autels ${ }^{\mathrm{I}}$ permet d'envisager combien leur opérativité dans le système d'objets du christianisme est fondamentalement liée au mystère de l'Incarnation. A la différence des images qui, pour le milieu carolingien notamment ${ }^{2}$, ne pouvaient être supports de passage (transitus) vers le prototype qu'elles figuraient 3 , la réitération du martyre du Christ à travers les corps des saints (Biotti-Mache, 2007) fait des reliques des véhicules privilégiés.

Entre l'ostension des reliquaires sur les autels dont témoigne le document exceptionnel ordonnant cette monstration pour le trésor de la cathédrale de Bâle ${ }^{4}$ et l'apparition des retables, on peut situer celle de premiers dispositifs de soclages, vers le $\mathrm{XI}^{\mathrm{e}}$ siècle, destinés à rehausser la visibilité des reliques et à prendre rapidement la forme de véritables structures verticales, supports d'une imagerie complexe autour de l'écrin des reliquaires (D'Hainaut-Zveny, 20I7, p. 24). L'autel majeur de Grandmont's et le retable de saint Remacle de

I. Sous le pontificat de Léon IV - 790-855, (Admonitio synodalis).

2. Aux prises avec les querelles iconoclastes dans l'Empire byzantin (Grabar, 1957).

3. Comme en témoigne la position restrictive des Libri Carolini (rédigés entre 79I et 794 sous la responsabilité d'Alcuin et de Théodulfe d'Orléans) contre l'iconolâtrie byzantine.

4. La représentation de l'ordre réglant la présentation des objets du Trésor sur l'autel majeur de la Cathédrale de Bâle au cours des jours de fêtes majeurs de la fin du Moyen Age a été donnée pour la première fois par Jacob Burckhardt en 1933, Die Kunstdenkmäler des Kantons Basel-Stadt 2, Der Basler Münsterschatz, Basel. On le retrouve notamment dans (Husband \& Chapuis, 20oI, p. I6, fig. 5) et dans (Mariaux, 2015).

5. Reconstitué par Jean-René Gaborit (1976). 
Stavelot ${ }^{\mathrm{I}}$ intégrant en son centre la châsse du saint en constituent les exemples les plus célèbres.

Dans quelle mesure l'apparition, dans l'Eglise du Moyen Age occidental, de ces dispositifs de mise en exposition (Mariaux, 2015) des matières sacrées sur les autels par le truchement de ces prémices de retables relève-t-elle des mécanismes de sacralisation précédemment évoqués? Y a-t-il à l'œuvre un processus de qualification topographique? Vraisemblablement oui, puisque l'autel majeur qui leur sert de support constitue l'épicentre du sanctuaire et peut ainsi fonctionner comme un « lieu autre » dont parle l'étymologie du sacré. Le retable occupe précisément un point le rendant lui-même susceptible d'opérer à la façon d'un seuil, à la fois borne et :

Frontière qui distingue et oppose deux mondes et le lieu paradoxal où ces mondes communiquent, où peut s'effectuer le passage du monde profane au monde sacré (Eliade, 1965, p. 28)

\section{9}

Quid d'une qualification temporelle? L'ostension des reliquaires sur le devant des autels interrompt le continuum du temps liturgique ordinaire qui les maintient dans le secret des trésors d'église (Ronig, I972; Sire, 200I). Le temps du pèlerinage et celui de l'exécution des rituels de circumambulation, qui en constituent souvent la dernière étape, constituent des formes supplémentaires de façonnement d'une «temporalité autre » autour de la rencontre avec les reliques ${ }^{2}$. L'adjonction de volets, sur certaines châsses 3 , puis aux côtés de triptyques-reliquaires ${ }^{4}$, va ensuite offrir un nouveau ressort essentiel au modelage de l'expérience temporelle de l'apparition.

\footnotetext{
I. Les dispositions de ce retable en argent doré sont connues à travers un dessin daté de I66I et conservé aux archives de l'Etat à Liège.

2. Le rôle éminemment actif du façonnement du temps entourant l'invention des reliques (inventio), leur installation dans des reliquaires ou des architectures (elevatio et translatio) et enfin leurs ostensions rituelles selon un calendrier précis constitue une part essentielle des évènements qui peuplent les chroniques des abbayes médiévales. Voir notamment les célèbres livres des miracles de sainte Foy de Conques (Liber Miraculorum Sanctae Fidis, 1994) ou encore des saints de l'abbaye de Savigny (Liber de Miraculis Sanctorum Savigniacensium, I243 [I899]).

3. En témoigne une gravure représentant la châsse des Rois Mages de Cologne, Le voyage de l'Empereur Henri, Trêves, vers I340, parchemin. Coblence, Staatarchiv, Ms. I C, n ${ }^{\circ}$ I, Balduineum. Gravure figurant dans le dossier iconographique annexe de la thèse de Brigitte d'Hainaut-Zveny déjá évoquée (2008).

4. Parmi les plus célèbres, datant du milieu du XII siècle, figurent celui provenant de Stavelot et conservé à la Pierpont Morgan Library de New York, celui conservé au Petit Palais et celui détenu par le Victoria and Albert Museum de Londres. Voir leur mention dans (Caillet, 2006).
} 
L'exemplaire attribué à Simone Dei Crucifissi i illustre la possibilité nouvelle de rehausser la préciosité des reliques enchâssées dans les cavités au pourtour du panneau central autant que d'en contrôler strictement la visibilité à travers le jeu de fermeture des volets latéraux. Le mouvement dialectique du caché/ dévoilé caractérisé plus haut par Schmitt devient ainsi le pivot de l'espérance épiphanique. Cette logique de privation visuelle procède à la fois du symbolisme de l'Incarnation et de l'attente de la Parousie manifestée pendant le Carême par le jeûne des images. Fondant également le contraste entre l'enclosure des reliques dans « la tour du trésor » (Roux, 20I0) et leurs ostensions aux tribunes, elle s'accentue encore dans les polyptyques-reliquaires de la sphère germanique ${ }^{2}$ du milieu du XIV siècle. Dans l'exemple du retable créé entre I340 et I360 pour l'église Sainte-Claire du couvent des Clarisses', la double articulation des volets permettait de réserver aux plus grandes solennités l'apparition des douze compagnes d'Ursule arborant des reliques par les ouvertures circulaires quadrilobées ajourant leurs poitrines. Un tel dispositif de régulation de la visibilité témoigne d'une transformation profonde des modes d'appréhension du sacré. On s'en rendra mieux compte en concevant, grâce à Alphonse Dupront, la primauté de la pulsion instinctive de contact charnel dans la gestuelle d'oraison liée aux reliques.

Toucher de la main ou avec un chapelet ou baiser le rocher de l'apparition, poser ses lèvres sur le reliquaire, frotter délicatement le tombeau, frôler du bout des doigts le socle sur lequel repose l'image ou encore pratiquer l'« abrazo » comme à Saint-Jacques-de-Compostelle, demander à un clergeron de mettre en contact avec la statue du saint des rubans dont on gardera une partie précieusement sur soi, autant de gestes qui marquent la rencontre, ce moment où le pélerin reçoit sa charge sacrale, promesse et puissance de la mutation (Dupront, 1987, p. 398-399).

Le sens très concret de la communion des saints des premiers siècles du christianisme (Vauchez, 2008) avait perduré pendant les siècles centraux du Moyen Age jetant les pèlerins au pied des châsses ou statues-reliquaires contre les flancs desquelles ils tombaient en oraison. L'articulation des volets des retables participe d'une évolution conduisant, dans le courant du XIII siècle, à l'apparition de dispositifs qui tout en se donnant comme « monstrances »- eucharistiques ou reliquaires (Dumoutet, I926 ; Tixier, 20I4)-opéraient avant tout comme mises à distance des objets du transitus. L'union croissante du voir et du croire

\footnotetext{
I. Triptyque-reliquaire daté des environs 1360 et conservé au Musée du Louvre.

2. Voir aussi le retable monumental de sainte Ursule dans l'abbaye cistercienne de Marienstatt et le Retable d'or de l'église d'Oberwesel.

3. Retable conservé dans la cathédrale de Cologne.
} 
pointée par Recht (1999), cristallisée dans l'instauration du rite de l'élévation de l'hostie rendue ainsi plus visible par les fidèles4 (Dumoutet, 1926; Tixier, 2014) traduit ainsi également une volonté de favoriser l'avènement d'une relation au sacré amenée à prendre la forme d'une communion plus spirituelles. Cette évolution conduira, au-delà du XIV ${ }^{e}$ siècle, à la transformation du statut des images avec l'entrée dans l'âge gothique (Bernazzani, 20I2 ; D'Hainaut-Zveny, 2008) et à la transformation du retable en « une ouverture vers un séjour qui ne sera jamais franchi » (Chastel, [1993] 2002, p 39). La métamorphose des voies d'accès à la transcendance passe ainsi par une mise à distance du corps à travers lequel le fidèle s'engageait précédemment tout entier au contact du sacré ${ }^{6}$ au profit d'une expérience plus intérieure vécue presque exclusivement à partir de perceptions optiques?. Comprenons ainsi que c'est bien au cour de l'évolution liturgique de la fin du Moyen Age (et non dans les effets du collectionnisme ou de la muséalité) qu'advient la transmutation de la façon dont les retables permettaient aux fidèles les contemplant de se lier aux mystères théologiques sur lesquels ils ouvraient.

\section{Le retable comme tableau}

Qu'il ait la simplicité monolithique des premiers spécimens sculptés dans un bloc de pierre ou de bois, inclue des éléments de prédelle, de fronton, de panneaux latéraux ou qu'il se complique pour présenter, autour du $\mathrm{XV}^{\mathrm{e}}$ siècle, des arcatures, bandeaux sculptés et nombreux pinacles, le retable constitue une unité dans l'espace du sanctuaire chrétien. Au plan formel, son organicité est liée à l'ordonnancement architectural du chœur, à l'articulation à la lumière ainsi qu'à la distribution des représentations iconographiques qui l'environnent et dont il est porteur (Chastel, I965, p. 242). Au-delà de son existence comme cellule artistique interdépendante, ce sont les relations qu'il noue avec les réalités supérieures qui fondent son unité et son efficacité. On peut dès lors supposer une corrélation entre les démembrements qui affectèrent les retables, dès le début du XVI ${ }^{\mathrm{e}}$ siècle, impliquant souvent la destruction de leurs cadres

\footnotetext{
4. Le IVe concile du Latran (I2I5) réaffirme la centralité du dogme de la transsubstantiation tandis qu’en I264 est instaurée la fêtte du «Corpus Christi » allant dans le sens de cette évolution de la dévotion.

5. La modification de la célébration eucharistique promue par Latran IV instaure une communion visuelle destinée à supplanter la communion sacramentelle que le concile limite à une seule fois par an.

6. Les pratiques dévotionnelles et les formes matérielles des objets ne se plient pas à des scansions si tranchées obéissant à des découpages chronologiques simplistes. Elles tendent bien plus à coexister et à «se chevaucher» entre elles qu’à se succéder de façon mécanique ou linéaire.

7. La piste suggérée par le professeur Pierre-Alain Mariaux de penser le glissement du tactile vers le visuel à travers la catégorie d'« haptique » qu'Aloïs Riegl avait forgée, dès I9or, à partir du grec grec aptô (toucher) donnant le terme d'“haptisch" dans L'industrie d'art romaine tardive (Die Spätromische Kunstindustrie) doit encore être explorée. De même pour le passage de l'haptique vers l'optique chez Riegl repensé par Deleuze proposant la notion de « vision haptique » pour dépasser la dualité du tactile et de l'optique. (Voir Bacon, 2002, p. 79-86).
} 
sculptés, et l'évolution liturgique déplaçant, après la Réforme, la focalisation sacrale des reliques et des images vers les espèces eucharistiques elles-mêmes. Dans cette perspective, l'invention « d'œuvres indépendantes » à partir de fragments circulant sur le marché de l'art de l'époque (Goldthwaite, I993) découle, au moins en partie, d'une forme d'affaiblissement de la cohésion sacrale structurant l'objet. Cette hypothèse vient compléter l'analyse faite ci-dessous par Chastel du glissement du retable du cultuel vers le culturel.

La déstructuration abolit l'existence liturgique du panneau pour le faire entrer dans le domaine de la «curiosité ». Les fragments des pale démontées ne deviennent pas seulement des tableaux, en ce sens que le regard de l'amateur ne s'attache plus qu'à leur style, dans d'innombrables cas, cette "métamorphose »- pour garder le terme de Malraux - a changé radicalement leur statut (Chastel, 1993, p. 20).

Cette assertion pointe plusieurs choses essentielles. Tout d'abord que l'histoire du tableau d'autel épinglé sur une cimaise comme le papillon dans la boîte d'entomologie ne commence pas si tard qu'on tend parfois à le croire. Le rappel de l'âge précoce du démembrement, situé ici au XVI siècle, est crucial pour établir que, dans le voyage des retables du sanctuaire vers le musée, ce dernier n'est pas l'acteur principal des transformations qu'on lui impute parfois hâtivement. La dispersion des panneaux devenus tableaux précède le musée d'art occidental et sa logique encyclopédiste de classement en spécimens. Elle s'inscrit dans un changement du regard sur ces œuvres antérieur aux Lumières. Où prend donc racine cette métamorphose ayant doté les œuvres, pour ellesmêmes, d'un prestige exceptionnel ? On se propose de montrer, ci-après qu'une part de cette transformation s'ancre dans le terreau de la Renaissance italienne. Elle sous-tend les réflexions que l'érudit Paulo Manuzio adresse, vers I56o, à Andrea Loredan après avoir visité sa collection comptant parmi les plus riches du XVI ${ }^{e}$ siècle vénitien :

Vous ne laisserez jamais [à vos fils] aucune propriété, aucun palais, aucun trésor qui puisse égaler la valeur et l'excellence de vos antiquités. Ce ne sont ni des biens matériels qu'on acquiert avec un simple effort ni des gemmes qu'on obtient par l'achat; ce sont des richesses vertueuses qui n'intéressent pas le vulgaire, mais qu’on réunit seulement grâce au jugement, à lintelligence, à une science infinie pendant un long espace de temps. Elles donneront aux siécles futurs un clair témoignage de la beauté de votre âme et de la noblesse de vos pensées, et, grâce à elles votre maison sera visitée et honorée par les 
étrangers désireux de voir les choses rares et parfaites (Loredan, 1560, p. 73 cité par Pomian, 2003, p. 59).

On émet ici l'hypothèse que l'essentiel de ce qui fondera, plus tard, le référentiel de valeurs et de représentations constitutif de l'institution muséale est contenu en germe entre ces quelques lignes. Quoiqu'il ne s'agisse pas là de fragments de retables, mais d'un cabinet d'antiques, on retient cet exemple pour la spontanéité avec laquelle il exprime la nouveauté d'une relation aux objets naissant avec la sensibilité du XVI e siècle et qui n'ira qu'en s'amplifiant face aux peintures (Pomian, 2003). Peut-on reconnaître dans l'évocation des effets que cette lettre attribue là à la collection des indices d'une mutation du régime de valeur attribué aux ouvres ? Ces indices pourraient-ils être rapprochés des mécanismes au cœur de l'élaboration sacrale décrite en préambule?

Bien que collectés en tant que vestiges concrets rescapés de l'époque romaine, les objets évoqués sont avant tout décrits ici par leur immatérialité. La transmutation pointée entre «biens matériels et richesses vertueuses » met sur la voie d'un processus à travers lequel ces artefacts manifestent autre chose qu'eux-mêmes. Le collectionnisme transforme des réalités terrestres issues d'un chaos indéterminé en manifestations d'un ordre supérieur déterminé par l'intellection. Les gestes qui le fondent ne rappellent-ils pas la gestuelle sacrale de circonscription, au sein d'un univers amorphe, d'un espace à l'intérieur duquel tout signifie? La mention du cabinet d'antiques comme lieu de ce «qui n’intéresse pas le vulgaire » lui confère un statut à part attirant à lui le désir de ceux qui en sont écartés. Dans cette dynamique d'élaboration spatiale, l'existence d'une marge informe joue également un rôle actif. Plus encore que la présence des érudits, c'est l'exclusion des profanes, cristallisée ici dans la figure du vulgaire, qui rend possible la valorisation de cet espace comme «centre du monde » (Eliade, 1965). La perspective de la curiosité est consubstantielle à la reconnaissance de la valeur de la collection et des mythes dont elle est porteuse.

Sans doute est-ce parce qu'elle rassemble moins des objets que des reflets de réalités impalpables que la collection peut devenir espace de projection et être investie d'affects pouvant rappeler la pulsion sacrale évoquée plus haut. Que dévoile en tout premier lieu le microcosme composé par le goût du collectionneur? Glorifiant l'esprit humain comme siège du « jugement », de «l'intelligence » et de la «science infinie », la collection opère avant tout autre chose comme miroir de l'image de son propre créateur. Elle ouvre sur une dimension invisible en second lieu à travers sa capacité de négociation avec le temps. Que Paulo Manuzio se projette dès le seuil du cabinet $«$ dans le forum de Rome $»^{1}$ signifie-t-il pour autant que la collection agit comme conservatoire d'un passé en tant que réalité objective? Certainement non. Au jaillissement désordonné

I. Mentionné dans la suite de la citation. 
du cours du temps, le filtre de l'érudition humaniste oppose avec autorité une recréation savamment ordonnée susceptible de faire saillir les grands jalons de la création. Est-ce que ce sont d'abord les ouvres que l'écrin de la collection fait échapper par-là au péril de l'inéluctable disparition? Avant elles, le collectionnisme célèbre et immortalise les sources de son savoir comme on l'entrevoyait à l'instant. Construction mémorielle, il affirme également l'autorité de ses propres représentations. A travers tout cela, indubitablement c'est la figure même du collectionneur, démiurge réordonnant le monde selon ce que lui dicte la sûreté de son goût, qui échappe la première au drame de la finitude.

Tels sont, d'après ce que peut permettre d'en dire ce fragment épistolaire, les mondes invisibles sur lesquels ouvre l'espace de la collection façonné par l'œil d'un humanisme dont la principale invention fut sans doute celle du génie humain. En même temps qu'il reçoit en partage les objets du collectionnisme de la Renaissance, le musée hérite de son système de représentation dont les fondements se situent bien plus puissamment du côté du mythe que de celui des objets qui l'actualisent. La prise en compte de ce basculement essentiel du rapport dévotionnel vers l'appréciation esthétique est indispensable pour comprendre le changement de paradigme que Pomian pointe, ci-après, au sujet du regard posé sur les œuvres au XVIII ${ }^{e}$ siècle.

Si on se réjouit maintenant que les étrangers viennent à Venise pour y admirer les tableaux - et non, comme jadis pour y adorer les reliques - c'est parce que à Venise, comme ailleurs en Europe, se propage parmi les élites une sorte de religiosité anthropocentrique qui voit dans l'Art - avec majuscule - l'expression privilégiée de la puissance créatrice de l'homme et place les ouvres d'art au sommet de la hiérarchie des productions humaines, leur conférant ainsi un statut plus élevé que jamais (Pomian, 2003, p. 99).

La centralité du retable sur l'autel du sanctuaire médiéval, dans son érection au statut de chef-d'œuvre à la gloire du génie dans la collection Renaissance comme dans l'espace des grandes galeries européennes, pourrait enfin avoir trait à sa propension à offrir un support à réalités invisibles. L'étymologie de la pala d'altare renvoyant littéralement « ce qui est visible, évident » (Blanc, 1998, p. 49) invite à examiner cet objet prioritairement à travers les investissements sensibles et symboliques que chaque époque a projetés sur lui. L'aborder dans sa dimension d'« objet transitionnel » au sens où l'a défini Winnicott (20ro), comme le fait l'essai Des retables et des hommes (D'Hainaut-Zveny, 20I7) consiste à saisir combien, loin de refléter passivement la présence divine au Moyen Age, ou le génie à la Renaissance, ce dispositif à la fois image, objet (Baschet, 2008) et écran porte les marques de l'imaginaire et des besoins de chaque temps et 
ce y compris dans le musée actuel. Le constat établi par Malraux peut alors être médité à nouveau.

Le musée arracha leur fonction aux ceuvres d'art quil réunissait, ne connut plus palladium, ni saint, ni Christ, ni objet de vénération [...], mais des images de choses différentes des choses elles-mêmes (Malraux, 2949, p. 15).

\section{9}

L'appropriation muséale des œuvres jadis chargées de numinosité constitue effectivement un processus de réélaboration. Au sein de ce processus, les glissements de valeurs, les traductions sémantiques, comme les formes d'amputation symbolique du pouvoir anciennement attaché à ces objets doivent être mis en lumière. L'adoption d'une posture anthropologique, encouragée par Bruno Brulon Soares (2018), peut contribuer à lever le voile (comme y invite également Ames (1994/1995)) sur les ressorts sous-tendant la réapparition, dans l'espace muséal, des artefacts supports de l'apparition du sacré. Une perspective qui conduit à sonder si l'auréole environnant la Vierge aux rochers, pour s'en tenir à cet exemple, ne résulte pas d'un système de croyances, de représentations, de discours et de dogmes tout aussi construit et puissamment agissant que celui sous-jacent à l'éblouissement provoqué précédemment par l'apparition épiphanique de l'or des retables comme des reliquaires dans l'écrin du sanctuaire?

\section{Conclusion}

Evoquer l'existence du retable dans l'environnement du sanctuaire avant de considérer son glissement vers les cimaises muséales a permis de s'interroger sur l'origine et la nature de la suspension d'un objet jadis façonné pour tisser un lien entre terre et Ciel. Ce parcours diachronique a également souligné combien la signification et le rayonnement de ces œuvres dépassaient les seuls dispositifs, actualisant leur présentation - cimaise ou autel. La perfection formelle des Vierges sur leurs trônes de gloire, des cohortes de saints en « conversation sacrée », comme des Descentes de croix existe presque dès l'instant de la commande des retables. La conscience de la valeur patrimoniale, comme de celle des objets des trésors d'église, est attestée par leur place dans les inventaires dès le début du Moyen Age. Mais aucune séparation n'existe alors entre ces valeurs et les pratiques rituelles, les formes de dévotion et les espérances attachées à ces créations liées à la matérialisation du sacré. Le raffinement des ordres superposés de sculptures sous les fines arcatures gothiques comme le resplendissement des dorures à l'or sont faits pour manifester la splendeur divine.

L'institution muséale est-elle en mesure de se porter garante aussi bien de la préservation matérielle de ce type d'objets que de la transmission du réseau hétérogène des raisons et significations multiples ayant fondé leur apparition? Envisagée ici face au corpus de supports du culte chrétien, ce questionnement 
peut être étendu à d'autres catégories d'objets investis de sacralité et dissociés de leur contexte d'usage premier et soulevant la même la question du devenir du « sacré des autres » (De L'Estoile, 2007).

Ecrivant "Take the crucifix out of the cathedral and you take the cathedral out of the crucifix" (I99I, p. 19), Philip Fisher pointe l'impossibilité de simplement renouer les attaches entre l'objet et son contexte d'origine. Il demeure cependant possible d'aspirer à l'invention d'une anthropologie des pratiques susceptible d'éclairer leurs appropriations successives par différentes cultures et combien celle-ci a, dans les différentes énonciations, partie liée avec les processus au cœur de l'élaboration sacrale. Ecran projectif de réalités immatérielles telles que la présence du sacré dans l'espace du sanctuaire, le retable offre un espace idéal pour cette investigation. Son examen révèle la part de symbolisation dans le processus de muséalisation. Déchirure et déni des investissements symboliques anciennement attachés à ce type d'objet, elle est aussi transfert et réinvention de leur valeur. Rapprochement enfin des fragments de tesselles brisés à l'origine de l'étymologie du symbole, elle peut encore être le lieu d'un rejaillissement de la numinosité des œuvres sous la forme de « l'aura » de Benjamin ( (I935 [1955]) ou sous la forme de la tentation de l'institution de se parer de ce rayonnement à travers le jeu constant de sa référence analogique au sacré (Heinich, 20I2). Si entre l'espace muséal et l'espace ecclésial des «parfums, [d]es couleurs et [d] es sons se répondent », c'est peut-être que, dans ces lieux « autres » (Foucault, I984), affleurent des quêtes parallèles face à la conscience de l'incomplétude, à la menace de la disparition.

Pour que l'affleurement du sacré au musée soit source d'une élévation comme le présuppose ce terme, on ne peut qu'aspirer, avec Stephan Greenblatt, voir se réaliser dans ses espaces ce qu'il a désigné comme la « résonnance » (Greenblatt, 199I). Alors, les dispositifs muséaux permettraient de communiquer les savoirs sur les strates antérieures de significations des objets, d'indiquer ce qui en eux ouvre sur la transcendance et enfin d'évoquer les dynamiques complexes à l'origine de leur intégration dans la sphère muséale. Seule l'exigence d'accueil à la fois de l'épaisseur historique des objets vécus comme numineux et de la sinuosité des voies de leurs réinterprétations successives peut faire de l'espace d'exposition un espace de résonnance à même d'offrir à l'homme qui le pénètre l'expérience de quelques « correspondances».

\section{Références}

Ames, M. (1994/1995). The Nature of Numinosity and its Museological Reconstruction. The Journal of the Walters Art Gallery, 52/53, 6I-64.

Bacon, F. (2002). La logique de la sensation. Paris, France : Editions La Différence. Baschet, J. (2008). Liconographie médiévale. Paris, France: Gallimard. 
Benjamin, W. (1935 [1955]). L'oeuvre d'art à l'époque de sa reproductibilité technique. Paris, France : Folio. (traduction Maurice de Gandillac).

Bernazzani, A. (2012). L'hostie et le retable : la contemplation comme rite de passage vers Dieu à la fin du Moyen Age. Acta Iassyensia Comparationis, 10, Rituri de Trecere/Rites of Passage/Rites de passage, 30-4I.

Biotti-Mache, F. (2007). Aperçu sur les reliques chrétiennes. Études sur la mort, I, I3I, II5-I32.

Blanc, M. (1998). Retables. La collection du musée des Arts Décoratifs. Paris, France : RMN.

Boskovits, M. (1992). Appunti per una storia délia tavola d'altare le origini. Arte cristiana, 80, 422-438.

Bousquet, J. (1982). Des antependiums aux retables. Le problème du décor des autels et de son emplacement. Cahiers de Saint-Michel de Cuxa, I3, 20I-232,

Brulon Soares, B. (20I8). Every museum has a God, or God is in every museum? In F. Mairesse (Ed.), Museology and the Sacred (pp. 44-48). Paris, France : ICOFOM.

Caillet, J-P. (2006). De l'antependium au retable : la contribution des orfèvres et émailleurs d'Occident. Cahiers de civilisation médiévale, I93, Janvier-mars 2006 (La médiévistique au XX $X^{\mathrm{e}}$ siècle. Bilan et perspectives.), 3-20.

Chastel, A. (1965). Le grand atelier d'Italie (1460-1500). Paris : Gallimard.

Chastel, A. (1993). La Pala ou le retable italien des origines à 1500. Paris, France : Liana Levi.

Chastel, A. ([1993], 2002). Histoire du retable italien des origines à 1500. Paris, France : Liana Levi.

D'Hainaut-Zveny, B. (2008). Les retables d'autels gothiques sculptés dans anciens les Pays-Bas. Raisons, Formes et Usages. Bruxelles, Belgique : Académie royale des Sciences, des Lettres et Beaux-Arts de Belgique.

D'Hainaut-Zveny, B. (2017). Des retables et des hommes. Diversité des objets mobilisés pour établir une relation avec le sacré dans les sanctuaires d'Occident (XIII ${ }^{\mathrm{e}}$-XVIII ${ }^{\mathrm{e}}$ siécles). Bruxelles, Belgique : Académie Royale de Belgique.

De L'Estoile, B. (2007). Le Goût des Autres. De l'Exposition coloniale aux Arts premiers. Paris, France : Flammarion.

Dumoutet, E. (1926). Le désir de voir l'hostie et la dévotion au Saint Sacrement. Paris, France : Beauschêne.

Dupront, A. (1987). Du sacré : croisades et pélerinages, images et langages. Paris, France : Gallimard.

Eliade, M. (1965). Le sacré et le profane. Paris, France : Gallimard. 
Fisher, P. (1991). Making and Effacing Art: Modern American Art in a Culture of Museums. Oxford, UK: University Press.

Foucault, M. (1984). Des espaces autres. Architecture, Mouvement, Continuité, 5 , octobre $1984,46-49$.

Fuchss, V. (1999). Das Altarensemble. Eine Analyse des Kompositcharakters früh und hochmittelalterlicher Altaraustattung. Weimar, Germany : VDG.

Gaborit, J. -R. (I976). L'autel majeur de Grandmont. Cahiers de civilisation médiévale, XIX, 23-246.

Gardner, J. (1994). Altars, Altarpieces and Art History Legislation and Usage. Dans E. Borsook et F. Superbi Goffredi, Italian Altarpieces, 1250-1550. Function and Design (pp. 5-19), Oxford, UK: Oxford University Press.

Goldthwaite, R. A. (1993). Wealth and the Demand for Art in Italy 1300-1600. Baltimore \& Londres: The Johns Hopkins University Press.

Gonseth, M.-O., Hainard, J., \& Kaehr, R (Eds.). (2002). Le musée cannibale. Neuchâtel, Suisse : MEN.

Grabar, A. (1957). L'iconoclasme byzantin. Dossier archéologique. Paris, France : Collège de France.

Greenblatt, S. (I99I). Resonance and wonder. In I. Karp \& S. D. Lavine (Eds.), Exhibiting cultures: the poetics and politics of museum display (pp. 43-56). Washington, USA; London, UK: Smithsonian Institution Press.

Grün, A. (2004). Entdecke das Heilige. Münsterschwarzach Abtei: Vier-Türme-Verlag.

Heinich, N. (2012). Des limites de l'analogie religieuse. Archives de sciences sociales des religions, 158-2012 Varia, I57-I77.

Husband, T., \& Chapuis, J.-C.(200I). The Treasury of Basel Cathedral. New York, USA: MET Publications.

Laclotte, M., \& Thiébaud, D. (I990). Polyptyques. Le tableau multiple du Moyen Âge au vingtiéme siècle [catalogue d'exposition, Musée du Louvre]. Paris, France : RMN.

Liber de Miraculis Sanctorum Savigniacensium. (I243 [1899]). Le livre des miracles des saints de Savigny: d'après le manuscrit original contemporain du roi saint Louis et composé aux années 1243 et 1244, traduit pour la premiere fois, avec préambule, par Hippolyte Sauvage.

Liber Miraculorum Sanctae Fidis. Recueil de textes latins écrits entre le XIe et le XIV siècle, reliés dans le manuscrit ms. 22 de la bibliothèque de Sélestat. i2o feuillets de parchemin. (I994). In L. A. d. 1. b. d. Sélestat (Ed.), Livre des miracles de sainte Foy. 1094-1994. Obernai. 
Mairesse, F. (2014). Le culte des musées. Bruxelles, Belgique : Académie royale de Belgique.

Malraux, A. (1949). Le musée imaginaire. Genève : Albert Skira Editeur.

Mariaux, P.-A. (2015). Exposer au Moyen Age? Le cas du trésor. In W. Brückle, P.-A. Mariaux \& D. Mondini (Eds.), Musealisierung mittelalterlicher Kunst (pp. 30-45). Berlin, Deutschland: Deutscher Kunstverlag.

Otto, R. (I968 [ì̀re éd. I9I7]). Le sacré : l'élément non-rationnel dans lidée du divin et sa relation avec le rationnel [trad. française par André Jundt de Das Heilige. Über das Irrationale in der Idee des Göttlichen und sein Verhältnis zum Rationalen. Paris: Payot.

Pomian, K. (2003). Des saintes reliques à l'art moderne. Venise-Chicago XIII'-XX siecle. Paris, France : NRF Gallimard.

Recht, R. (I999). Le croire et le voir. L'art des cathédrales (XII $-X V^{\mathrm{e}}$ siécle). Paris, France : Gallimard.

Ronig, F. J. (1972). Trésors et chambres des reliques Rhin-Meuse (Vol. volume I, pp. I34-I4I).

Roux, B. (2010). La tour du trésor : clés d'accès. In P.-A. Mariaux, L. Burkart, Y. Pottin, \& P. Cordez (Eds.), Le Trésor au Moyen Age : discours, pratiques et objets, études réunies par P-A Mariaux, L. Burkart, Y. Pottin, P. Cordez \& al. (pp. 89-Iог). Florence: Sismel Edizioni.

Schmitt, J.-C. (I997). Les reliques et les images. In E. Bozoky \& A.-M. Helvétius (Eds.), Les reliques. Objets, cultes, symboles. Actes du colloque international de l'Université du Littoral-Côte-d'Opale (pp. I45-I67). Boulogne-sur-Mer, France : Brepols. Hagiologia Atelier belge d'études sur la sainteté en Occident.

Sire, M.-A. (200I). Les trésors de cathédrales : salles fortes, chambres aux reliques ou cabinets de curiosité ? In J. s. 1. p. d. Le Goff, C. s. 1. d. d. Arminjon, \& D. Lavalle (Eds.), zo siécles en cathédrales (pp. I9I-202). Paris, France : Centre des Monuments Nationaux, Monum Editions du Patrimoine.

Tixier, F. (2014). La monstrance eucharistique. Genése, typologie et fonctions d'un objet d'orfevrerie (XIII'-XVI siécle). Rennes, France : Presses Universitaires de Rennes.

H. Van Os et al., Sienese Altarpieces, 1215-146o. Form, Content, Function, 2 vol. (Medievalia Groningana, VIII IX), Groningue, I988/90

Vauchez, A. (2008). Du culte des reliques à celui du Précieux Sang. Tabularia "Études ", nº 8, décembre, 8I-88.

Winnicott, D. W. (2010). Les objets transitionnels. Paris, France : Payot. 